\title{
Infected Mesh Removed After 8 Years of Inguinal Hernioplasty... Could Honey be the Rescuer?: A Case Report
}

\author{
Rajesh Chaudhary ${ }^{1}$, Ramesh Bharti' ${ }^{2}$, Amar Verma ${ }^{3}$, Rajesh Sharma ${ }^{2}$, Amit Dogra ${ }^{4}$, Ravi Singh Dogra ${ }^{4}$ \\ ${ }^{1}$ Senior Resident, Department Of Surgery, Dr. R.P. Govt. Medical College Kangra at Tanda HP, India, ${ }^{2}$ Professor, Department Of Surgery, Zonal Hospital \\ Dharamshala HP, India, ${ }^{3}$ Associate Professor, Department Of Surgey, Dr. R.P. Govt. Medical College Kangra at Tanda HP, India, ${ }^{4}$ Junior Resident, \\ Department Of Surgery, Dr. R.P. Govt. Medical College Kangra at Tanda HP, India.
}

\section{Abstract}

With the ever increasing number of Lichtenstein's tension free mesh inguinal hernia surgeries being performed worldwide, we are going to see an increasing number of deep mesh infections. In half of the cases the mesh can be saved with conservative treatment, but in other half it has to be removed ultimately to completely eradicate the groin sepsis. We had a patient with deep seated mesh infection presenting with a pus discharging sinus 8 years after Lichtenstein's tension free hernioplasty. The wound was explored and a piece of polypropylene mesh was removed. The infected wound which opened after few days of surgery was managed successfully with honey application only. In the modern era of antibiotic resistance honey is a boon to the human race which has been ignored for the reasons unknown.

Keywords: Infected mesh, SSI, honey, hernioplasty.

Corresponding Author: Dr. Rajesh Chaudhary, Senior Resident, Department Of Surgery, Dr. RP Govt. Medical College Kangra at Tanda HP, India.

Received: June 2019

Accepted: July 2019

\section{Introduction}

Inguinal hernia surgery has made great advancement in the last century. We have seen hernia surgery to evolve from a mutilating surgery which involved the compulsory removal of the testes, into the era of tissue repairs brought forward by Bassini and mastered by surgeons like Shouldice. Introduction of tension free hernioplasty by Lichtenstein, using polypropylene mesh to strengthen the posterior wall of the ingunal canal was a real game changer in the hernia surgery. ${ }^{[1]}$ Now a days hernia surgery is easy and safe to perform. The recurrence rate after tension free repairs has been reported to be less than $1 \%{ }^{\left[{ }^{2]}\right.}$ But during all these times the most serious complication of hernia surgery has been the surgical site infection (SSI) especially the deep seated mesh infections. The patients may present with local abscess, ulcers, sinus or fistulae. A mesh is a foreign substance inside the body which is bound to increase the ssi rate. It is not clear whether Antibiotic prophylaxis, mesh material, fixing material, prior skin infections increase the rate of deep seated mesh infections. It is possible to save half of these mesh with conservative methods like antibiotic and abscess drainage. ${ }^{[3]}$ Honey was in use for the treatment of wide variety of acute as well as chronic infected wounds since the ancient times. It has been used for the treatment of skin and soft tissue infections. It is a very good debriding agent which has antiseptic properties, at the same time it promotes healing. The advent of antibiotics has pushed this time tested remedy out of contention, but with the ever increasing incidence of multidrug resistant bacterias, it's very safe and effective to use honey for the wound management. ${ }^{[4]}$

\section{Case Report}

A 62 year old male presented to the surgical OPD (outpatient department) of our tertiary care center. Patient had an inguinal hernia for which he had undergone hernioplasty 8 years back at some private clinic. He remained asymptomatic for five years after surgery. Then he started having pus discharge from medial edge of the wound. He was managed initially with the help of antibiotics and local pus drainage for approximately 5 months. His condition persisted so his mesh was removed at the same clinic. He remained asymptomatic for 3 months and again started having pus discharge from the medial edge of the wound just below the incision scar. He was again put on conservative treatment but the condition persisted. He would remain asymptomatic as long as he took antibiotics, but the pus discharge again started once he stopped the antibiotics. He then came to our hospital. He had a pus discharging sinus just below the medial edge of inguinal incision [Figure 1]. Pus was subjected to culture and sensitivity which showed staphylococcus aureus. Pus was negative for acid fast bacilli. Patient was put on antibiotics for two weeks and an MRI (Magnetic Resonance Imaging) was planned. He was not a known diabetic and didn't have any other comorbities. MRI 
was suggestive of a sinus tract extending from the skin into the subfascial planes in the right inguinal region with a collection of about $2 \times 2 \mathrm{~cm}$ with multiple ramifications of the sinus tract. No communication with the bowel loops was seen. The patient was taken up for exploration under regional anaesthesia. On following the sinus tract during surgery a 7 $\mathrm{cm}$ long segment of the polypropylene mesh was removed along with the prolene sutures [Figure 2]. The pus was drained and wound closed after keeping in a suction drain. The drain was removed on third day. On 6th post-operative day the patient developed a surgical site infection following which the wound was opened and pus drained [Figure 3]. Pus culture revealed E.coli. Sensitive to cotrimoxazole. Even after seven days of the antibiotic therapy and daily ASD (antiseptic dressing) the wound didn't improve so honey dressing was started with commercially available honey. The wound showed good recovery and the patient was discharged home to continue the honey dressing after 10 days when the wound has contracted significantly [Figure 4]. Honey dressing was continued and the wound healed completely in a total time of about four weeks.

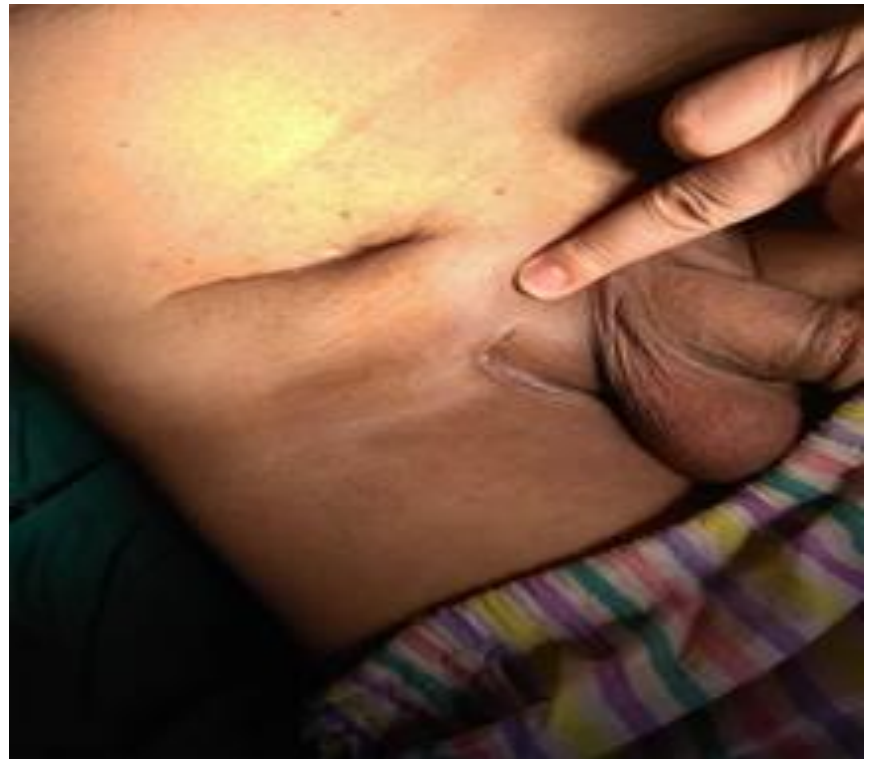

Figure 1: showing the infrainguinal sinus with the inguinal incision scar.

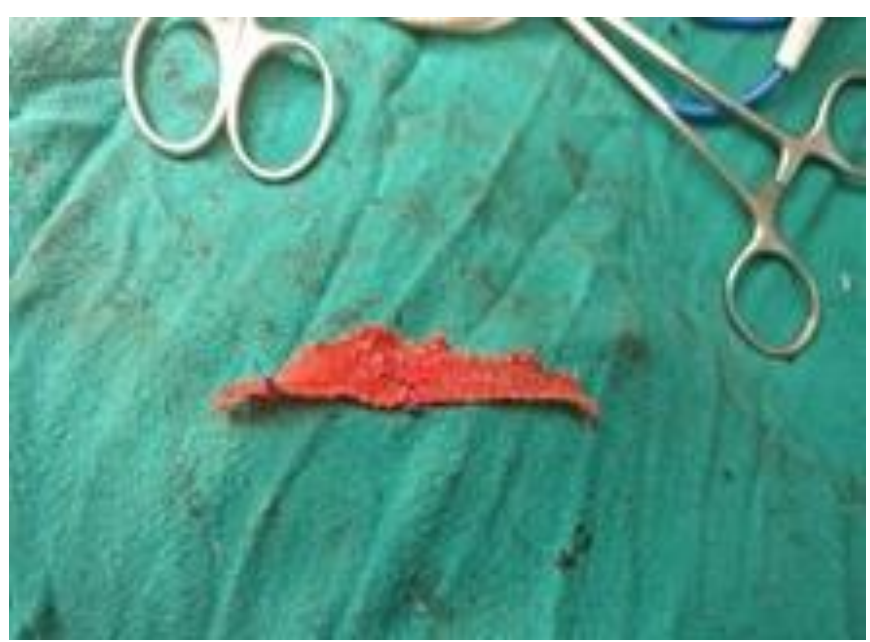

Figure 2: showing the removed part of mesh along with Prolene sutures.

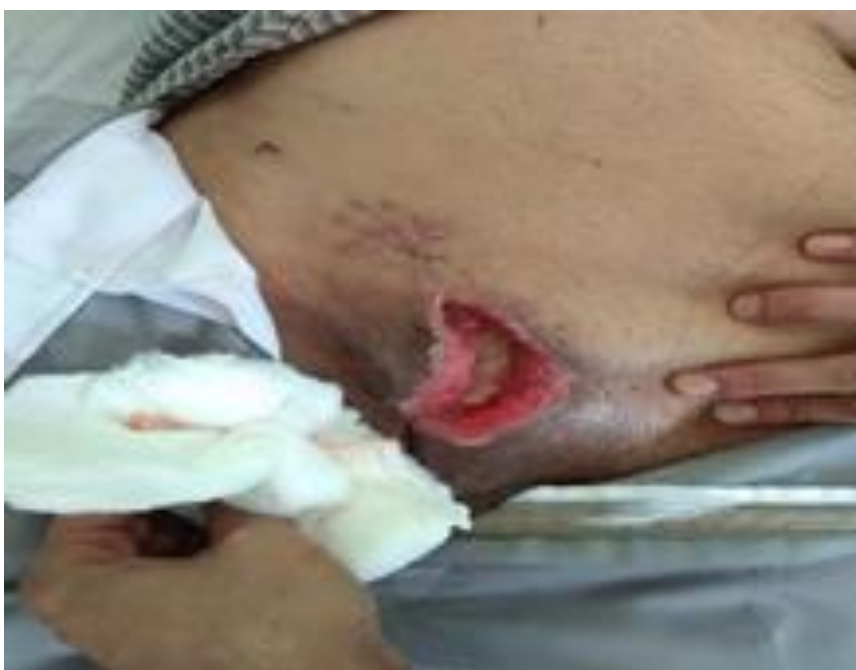

\section{Figure 3: showing deep SSI with dirty wound.}

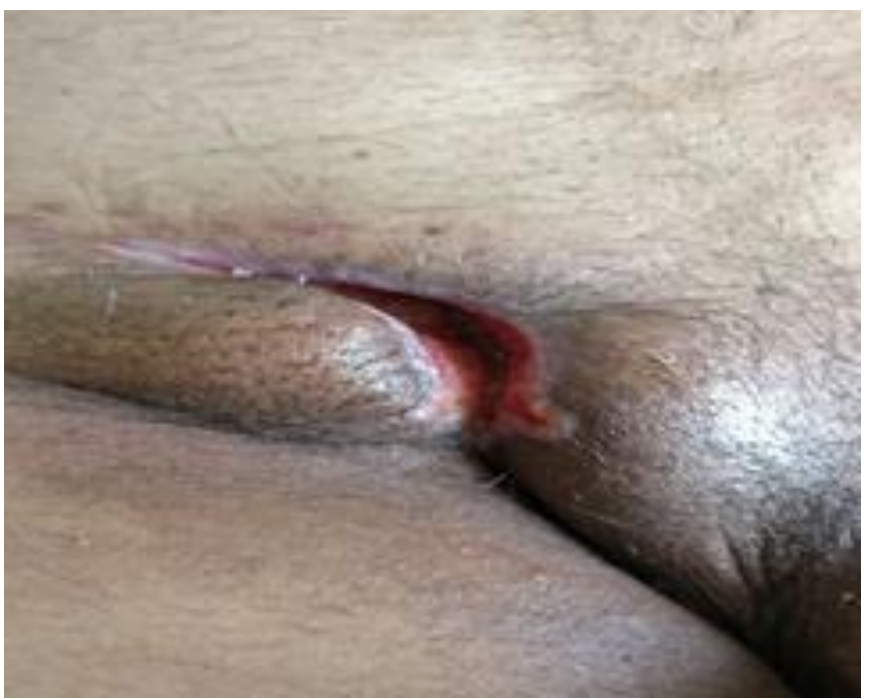

Figure 4: showing contracting healthy wound on day 7 of honey application.

\section{Discussion}

Inguinal hernioplasty is one of the most commonly performed surgeries worldwide by the general surgeons. There was a time when it was a mutilating surgery which usually sacrificed the testes of the involved side but bassini brought in the concept of tissue repairs to strengthen the ingunal canal. The tissue repairs were pioneered in the coming years but hernia surgery got revolutionised when Lichtenstein used the marlex mesh for strengthening the posterior wall of the inguinal canal. The recurrence rate was very low and it was easy to perform but the infection chances were high. ${ }^{[5]}$ The SSI rate after inguinal hernioplasty has been reported between $0-14 \%$, however the reported infection rate by Lichtenstein in his own series was $0.003 \%{ }^{[6]}$ Most of the infections are superficial SSIs which occur in the immediate post-operative period but the deep seated mesh infections, although very rare, are being reported too. The superficial SSIs are initially managed conservatively with the help of antibiotics and wound drainage and it is usually possible to salvage the mesh but deep infections causing the prolonged groin sepsis may 
require the removal of the infected mesh. Early infections are due to contamination of surgical field but the delayed deep seated mesh infections are thought to be due to persistent fluid collections. ${ }^{[3]}$ Most commonly associated pathogens are staph. Aureus, e.coli.,enterococci and coagulase negative staphylococci but pseudomonas aeuroginosa has also been reported in a patient after 14 years of hernioplasty, but the longest duration is 17 years after the surgery. Virulence of the bacteria, the wound microenvironment and the bacterial load are thought to be associated with the possible deep seated mesh infections but havent been proved confidently. ${ }^{[6]}$

Delikoukos et al has found no association between the use of prophylactic antibiotics and deep seated mesh infections. They said that the mesh material or the fixing materials also don't affect the SSI rate [3]. Diagnosis should be suspected in a patient with history of mesh implantation presenting with prolonged skin infections, recurrent local abscesses, sinuses or fistulae. Radiological investigations like ultrasonogram and contrast enhanced computerised tomogram (CECT) can help reach the diagnosis but they are neither always required, nor are they always fullproof. ${ }^{[7]}$ Initial treatment of the superficial as well as deep seated mesh infections is essentially conservative in the form of antibiotics and abscess drainage. With this management strategy about half of the meshes can be saved. Only those patients who donot respond well to conservative treatment or have frequent relapses will require the mesh removal. ${ }^{[8]}$ Honey could be a possible savior of the mesh in such cases. Honey is an easily available nature's gift to the humanity. In ancient times it was used for the treatment of diarrhoea, peptic ulcer disease, prevention of corneal ulcers and in measles. It has been used in the management of acute as well as chronic wounds. There has been studies confirming its efficacy and superiority in the management of burn wounds, venous ulcers, burst abdomen, pressure sores, amputation stumps, fournier's gangrene, diabetic foot and even chronic osteomyelitis.it is a natural debriding agent which not only removes the slough but also prevents it's further formation by promoting the autolysis brought on by activation of proteases. It releases a low concentration of hydrogen peroxide inside the wound which gives it the microbicidal properties. It promotes epithelisation of the tissues by withdrawing lymph within the wounds. ${ }^{[9]}$ With the ever increasing incidence of antimicrobial resistance it's very wise to give honey a chance to show it's efficacy in wound management. We haven't found any record of such case being reported in the past where honey was successfully used to treat a wound having deep seated mesh infection.

\section{Conclusion}

We will see a rise in the number of deep seated mesh infections in the near future with an ever increasing rate of hernioplasties being performed. The antibiotic resistance is a big menace, causing huge suffering to the patients as well as hospitals. All those mesh which will require removal after the failure of conventional conservative therapy for a variable time, are probable candidates where honey could be effective in salvaging the mesh. This opens up a new avenue for research in the management of complicated, deep seated mesh infections.

\section{Consent}

Written informed consent was obtained from the patient for publication of this Case report and any accompanying images. A copy of the written consent is available for review by the Editor-in-Chief of this journal.

\section{Author's Contributions}

Rajesh Chaudhary: Contributed substantially to the Conception, design, Acquisition of data, Analysis and interpretation of data, drafting the article, Critical revision of the article and final approval of the version to be published.

Rajesh Sharma: Contributed substantially to the Conception and design, Acquisition of data, Critical revision of the article and final approval of the version to be published

Amar Verma: Contributed substantially to the Conception and design of the article and final approval of the version to be published.

Amit Dogra: Contributed substantially to the Conception and design, Acquisition of data, Critical revision of the article and final approval of the version to be published.

Ramesh Bharti: Contributed to the Acquisition of data, Critical revision of the article and final approval of the version to be published.

Ravi Singh Dogra: Contributed substantially to the Conception and design, Acquisition of data, Critical revision of the article and final approval of the version to be published.

\section{References}

1. Hee RV. History of inguinal hernia repair. jurnalul de chirurgie, iaşi, 2011, vol. 7, nr. 3

2. Ersoz F, Culcu S, Duzkoylu Y, et al. The Comparison of Lichtenstein Procedure with and without Mesh-Fixation for Inguinal Hernia Repair. Surgery Research and Practice. 2016;2016:8041515. doi: $10.1155 / 2016 / 8041515$.

3. Delikoukos S, Tzovaras G, Liakou P, Mantzos F, HatzitheoWlou C. Late-onset deep mesh infection after inguinal hernia repair. Hernia (2007) 11:15-17 DOI 10.1007/s10029-006-0131-1

4. Chaudhary AR, Sharma S, Shukla A, Joshi A, Chaudhary UK (2015) Honey 'The Life Saviour' in NecrotisingFascitis: A Case Report. Dermatol Case Rep 1:102. doi:10.4172/DMCR.1000102

5. Brunicardi FC.(2010) Inguinal hernia .In: Schwartz SI and Brunicardi CF (Eds.), Schwartz Principles of Surgery. (9th edn.), McGraw-Hill Health Pub. Division, New York, USA, pp. 2514-2586.

6. Filippou D. Late Ps. aeruginosa Inguinal Mesh Infection 12 Years after the Initial Operation: Report of the Case and Short Review of the Literature. Case Reports in Surgery Volume 2017, Article ID 4385913, 4 pages https://doi.org/10.1155/2017/4385913

7. Akyol C, Kocaay F, Orozakunov E, Genc V, Bayram IK, Cakmak A, Baskan S, Kuterdem E. Outcome of the patients with chronic mesh infection following open inguinal hernia repair.jJ Korean surgsoc 2013; 84: 287- 291.

8. Montgomery A, Kallinowski F and Köckerling F (2016) Evidence for Replacement of an Infected Synthetic by a Biological Mesh in Abdominal Wall Hernia Repair. Front. Surg. 2:67. doi: 10.3389/fsurg.2015.00067

9. Molan, P.C. (2009). Debridement of wounds with honey. Journal of Wound Technology, 5, 12-17. 
Copyright: () the author(s), 2019. It is an open-access article distributed under the terms of the Creative Commons Attribution License (CC BY 4.0), which permits authors to retain ownership of the copyright for their content, and allow anyone to download, reuse, reprint, modify, distribute and/or copy the content as long as the original authors and source are cited.

How to cite this article: Chaudhary R, Bharti R, Verma A, Sharma R, Dogra A, Dogra RS. Infected Mesh Removed After 8 Years of Inguinal Hernioplasty... Could Honey be the Rescuer? : A Case Report.Acad. J Surg. 2019;2(1):47-50.

DOI: dx.doi.org/10.21276/ajs.2019.2.1.13

Source of Support: Nil, Conflict of Interest: None declared. 